\title{
Simulation of surgery for supratentorial gliomas in virtual reality using a 3D volume rendering technique: a poor man's neuronavigation
}

\author{
Jaskaran Singh Gosal, MCh, DNB,, Sarbesh Tiwari, MD, DM, ${ }^{2}$ Tarunesh Sharma, MS, ${ }^{1}$ \\ Mohit Agrawal, MCh, ${ }^{1}$ Mayank Garg, MCh, ${ }^{1}$ Sayani Mahal, MD, ${ }^{2}$ Suryanarayanan Bhaskar, MCh, ${ }^{1}$ \\ Raghavendra Kumar Sharma, MCh, ${ }^{1}$ Vikas Janu, MCh, ${ }^{1}$ and Deepak Kumar Jha, $\mathbf{M C h}^{1}$
}

Departments of ${ }^{1}$ Neurosurgery and ${ }^{2}$ Diagnostic \& Interventional Radiology, All India Institute of Medical Sciences (AllMS), Jodhpur, Rajasthan, India

\begin{abstract}
OBJECTIVE Different techniques of performing image-guided neurosurgery exist, namely, neuronavigation systems, intraoperative ultrasound, and intraoperative MRI, each with its limitations. Except for ultrasound, other methods are expensive. Three-dimensional virtual reconstruction and surgical simulation using 3D volume rendering (VR) is an economical and excellent technique for preoperative surgical planning and image-guided neurosurgery. In this article, the authors discuss several nuances of the 3D VR technique that have not yet been described.
\end{abstract}

METHODS The authors included 6 patients with supratentorial gliomas who underwent surgery between January 2019 and March 2021. Preoperative clinical data, including patient demographics, preoperative planning details (done using the VR technique), and intraoperative details, including relevant photos and videos, were collected. RadiAnt software was used for generating virtual 3D images using the VR technique on a computer running Microsoft Windows.

RESULTS The 3D VR technique assists in glioma surgery with a preoperative simulation of the skin incision and craniotomy, virtual cortical surface marking and navigation for deep-seated gliomas, preoperative visualization of morbid cortical surface and venous anatomy in surfacing gliomas, identifying the intervenous surgical corridor in both surfacing and deep-seated gliomas, and pre- and postoperative virtual 3D images highlighting the exact spatial geometric residual tumor location and extent of resection for low-grade gliomas (LGGs).

CONCLUSIONS Image-guided neurosurgery with the 3D VR technique using RadiAnt software is an economical, easyto-learn, and user-friendly method of simulating glioma surgery, especially in resource-constrained countries where expensive neuronavigation systems are not readily available. Apart from cortical sulci/gyri anatomy, FLAIR sequences are ideal for the 3D visualization of nonenhancing diffuse LGGs using the VR technique. In addition to cortical vessels (especially veins), contrast MRI sequences are perfect for the 3D visualization of contrast-enhancing high-grade gliomas.

https://thejns.org/doi/abs/10.3171/2021.5.FOCUS21236

KEYWORDS image-guided neurosurgery; volume rendering technique; 3D reconstruction; computer-assisted neurosurgical procedure; surgical simulation; virtual reality; glioma; RadiAnt

$\mathrm{T}$ HE concept of image-guided surgery was introduced in neurosurgery by accomplishing the first stereotactic procedure toward the middle of the last century. ${ }^{1,2}$ Subsequently, the use of intraoperative ultrasound and the application of neuronavigation systems in the early 1990s augmented image-guided neurosurgery. ${ }^{3-8}$ Intraoperative MRI, another imaging-guided technique, has now been proven in many randomized studies to improve gross-total resection and progression-free survival rates for patients with glioma. ${ }^{9-15}$ Image-guided neurosurgery is currently the standard technique for the excision of intracranial lesions. ${ }^{16-18}$ However, the aforementioned systems have their practical limitations, and, except for ultrasound, they are expensive. Most are still not widely available, especially in resource-constrained countries. ${ }^{19,20}$

The 3D volume-rendering (VR) technique allows large volumes of data generated by modern CT and MR scanners to be viewed in a 3D virtual space. ${ }^{21}$ This technique is now readily available as a tool in several DICOM viewer software programs available online, namely, OsiriX (www.osirix-viewer.com), Horos (www.horosproject.org), and RadiAnt (Medixant). ${ }^{19-25}$ The different aspects of the

ABBREVIATIONS HGG = high-grade glioma; $L G G$ = low-grade glioma; VR = volume rendering

SUBMITTED April 1, 2021. ACCEPTED May 18, 2021.

INCLUDE WHEN CITING DOI: 10.3171/2021.5.FOCUS21236. 
TABLE 1. Clinical features and the clinical application of 3D VR technique in our patients

\begin{tabular}{|c|c|c|c|c|c|c|}
\hline $\begin{array}{l}\text { Case } \\
\text { No. }\end{array}$ & $\begin{array}{l}\text { Age (yrs), } \\
\text { Sex }\end{array}$ & Diagnosis & EOR & $\begin{array}{l}\text { New } \\
\text { Deficits }\end{array}$ & Pathology & Clinical Utility of VR \\
\hline 1 & $37, M$ & $\begin{array}{l}\text { Rt posterior frontal } \\
\text { LGG }\end{array}$ & GTR & None & WHO grade II astrocytoma & Simulation of skin incision \& craniotomy \\
\hline 2 & $21, \mathrm{~F}$ & Lt peritrigonal LGG & GTR & None & Pilocytic astrocytoma (grade I) & Navigation for deep-seated glioma $4 \mathrm{~cm}$ from cortex \\
\hline 3 & $41, \mathrm{M}$ & Rt parietal LGG & NTR & None & $\begin{array}{l}\text { WHO grade II astrocytoma (gem- } \\
\text { istocytic) }\end{array}$ & $\begin{array}{l}\text { Preop 3D morbid cortical surface \& venous anatomy in } \\
\text { surfacing gliomas }\end{array}$ \\
\hline 4 & $50, \mathrm{~F}$ & $\begin{array}{l}\text { Rt posterior frontal } \\
\text { HGG }\end{array}$ & NTR & None & Glioblastoma (WHO grade IV) & $\begin{array}{l}\text { Identification of operative corridor btwn cortical veins } \\
\text { for surfacing glioma }\end{array}$ \\
\hline 5 & $33, \mathrm{M}$ & $\begin{array}{l}\text { Pineal region } \\
\text { tumor }\end{array}$ & NTR & None & Ependymoma (WHO grade II) & $\begin{array}{l}\text { Preop planning of the route \& trajectory of approach } \\
\text { based on intervenous corridor in subcortical gliomas }\end{array}$ \\
\hline 6 & $31, M$ & Lt temporal LGG & GTR & None & Oligodendroglioma (WHO grade II) & Pre- \& postop 3D spatial geometry of LGGs \\
\hline
\end{tabular}

EOR = extent of resection; GTR = gross-total resection; NTR = near-total resection; STR = subtotal resection.

standard CT/MRI data set can be interactively explored in the 3D VR window. Compared with other techniques, this technique (as a form of image-guided surgery) is inexpensive and readily available. ${ }^{20}$

The clinical application of the 3D reconstruction technique in neurosurgery was demonstrated as early as 1998. ${ }^{26}$ Since then, several series have described its clinical application in different subspecialties of neurosurgery, such as vascular, skull base, spine, and tumors. ${ }^{21,27-31}$ Authors of most of these articles have used OsiriX as the software for VR of the standard MR images. ${ }^{22,23,27,31-34}$ Despite their clinical value and easy availability, many of these software programs are still not popular among neurosurgeons because of the complex user interface and a long learning curve. ${ }^{32}$ Hence, these software programs continue to be used mainly by radiologists for diagnostic purposes.

In this article, we discuss the advantages of the RadiAnt DICOM viewer over other software programs for creating virtual 3D images using the VR technique, and we demonstrate different clinical applications of this technique in the surgical management of supratentorial gliomas via case illustrations with several nuances not discussed earlier in the literature.

\section{Methods}

A retrospective review of prospectively collected data was done at a tertiary care center. All patients with supratentorial gliomas who underwent surgery between January 2019 and March 2021 in which the VR technique was used for planning the surgical approach were included. Preoperative clinical data, including age, sex, clinical history, preoperative planning details, intraoperative details with photographs and videos, and postoperative data were collected. Cases included 1 deep-seated supratentorial pilocytic astrocytoma, 3 diffuse low-grade gliomas (LGGs; WHO grade II), 1 glioblastoma, and 1 pineal region tumor (ependymoma) (Table 1). Informed written consent, per departmental protocol at our center, was obtained from all patients. Since this is a retrospective review of clinical records at our hospital where the patient management was not altered, ethics clearance was not deemed necessary.

\section{Acquisition and Processing of Images}

MRI at our center was performed on a 3T Discovery MR750 scanner (General Electric). The standard MR sequences-T1-weighted spin echo, T2-weighted turbo spin echo, 3D FLAIR, T1-weighted, T2-weighted, and postcontrast (postgadolinium) T1-weighted sequences-were used to acquire the images. All imaging studies were obtained in digital versatile disc format and were viewed on a personal computer. We used RadiAnt software to load the MR images in DICOM format. The images were viewed in $2 \mathrm{D}$ and then converted into 3D using the VR technique.

\section{Requirements}

Basic requirements for obtaining 3D virtual volumerendered images are as follows: 1) DICOM viewer software with VR tool, RadiAnt, freely available for download on the internet; 2) CT and MR images in the DICOM format; and 3) a computer running Microsoft Windows 10 (Microsoft Corp.). Although we used RadiAnt software on the Windows operating system in our study, this software can also be run on the Macintosh operating system with the help of third-party software. RadiAnt has a single user-friendly toolbar that can be used for creating and editing the virtual 3D images. Earlier, we described the various options and the method of using the toolbar provided with this software. ${ }^{21}$ Video 1 demonstrates many of these tools and their usage in detail.

VIDEO 1. Demonstration of cortical surface anatomy and navigation for a deep-seated glioma using the 3D VR technique on RadiAnt software. Copyright Deepak Jha. Published with permission. Click here to view.

Briefly, there are two methods in the RadiAnt software by which structures deep to the bone and dura (cortical surface and venous anatomy and mass lesions such as gliomas) can be visualized using the VR technique. One is simply by adjusting the window (done either by pressing the mouse's scroll button and sliding it or clicking the "adjust window" icon in the toolbar). This method is straightforward and does the job in most situations. Another way is by using the cutting tool and virtually removing the scalp, bone, and dura in layers to visualize the underlying structures, such as cortical veins. 

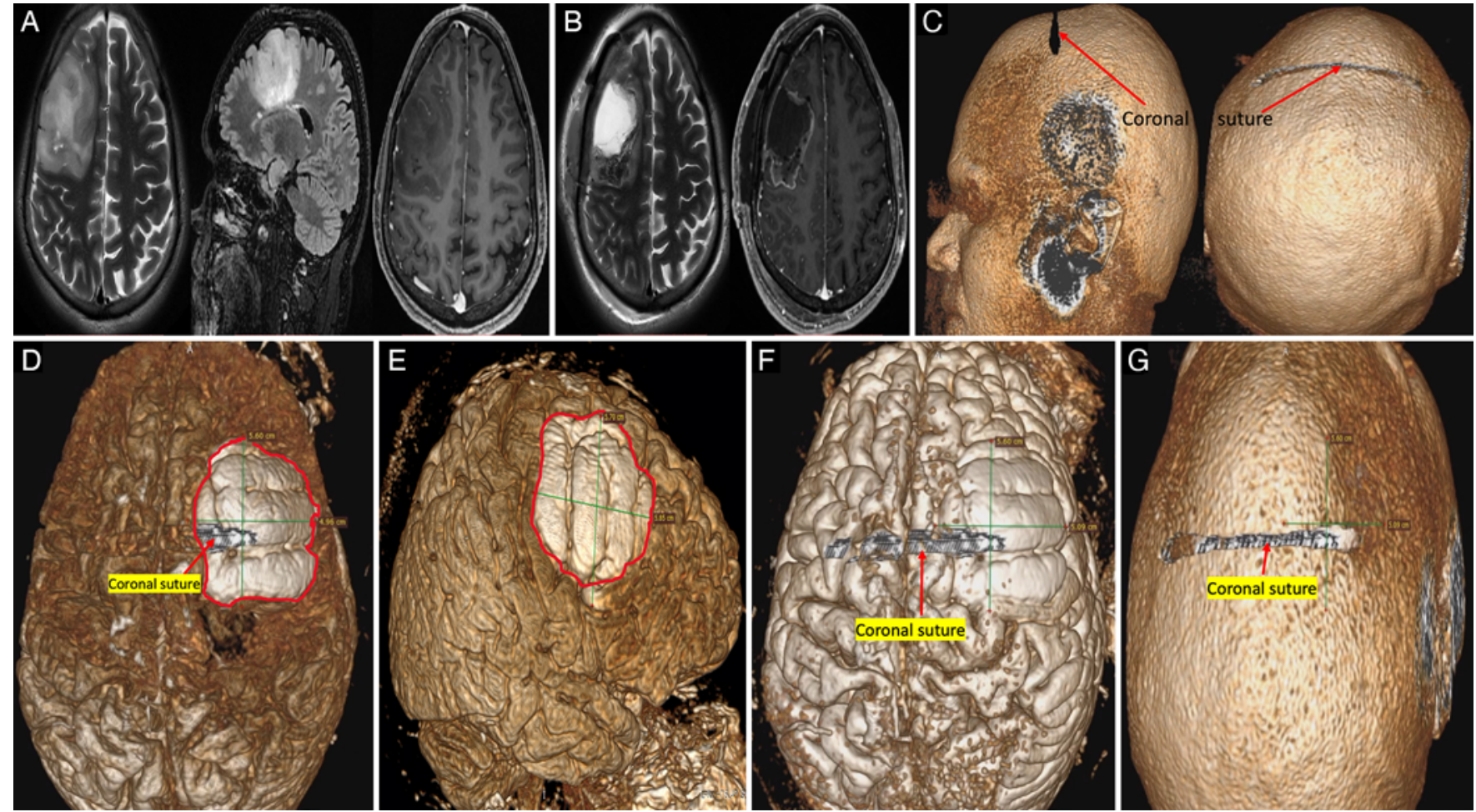

FIG. 1. Case 1. Imaging. A: Axial (left) T2-weighted, sagittal FLAIR (center), and contrast axial (right) MR images showing a right posterior frontal LGG. B: Postoperative axial T2-weighted (left) and contrast (right) MR images showing gross-total excision. Planning. C-G: A preoperative T2-weighted FLAIR MR image was reconstructed into 3D images using the VR technique. The coronal suture was marked over the skull by using the cutting tool (C), and, after adjusting the window, an already marked coronal suture was visible over the lesion ( $D$ and $F$ ). Expanded abnormal cortical gyri are appreciable ( $D$ and $E)$. The lesion's exact measurement, both anterior and posterior to the coronal suture, was appreciated ( $F$ and $G$ ).

\section{Optimal Sequences for Generating 3D VR Images}

It is essential to ensure that both the FLAIR and contrast MR images are provided in very thin (1-mm) contiguous nonoverlapping cuts, as these are the most important sequences for creating the virtual 3D images using the VR technique. 1) The T2-weighted FLAIR MRI sequence is converted into 3D VR images that show the cortical surface anatomy (sulci, gyri) in fine detail. This sequence is also ideal for the 3D visualization of nonenhancing diffuse grade II gliomas. 2) Conversion of the contrast MRI sequence into $3 \mathrm{D}$ images is best for demonstrating cortical vessels, including veins, as well as for the $3 \mathrm{D}$ visualization of contrast-enhancing lesions, such as high-grade gliomas (HGGs).

\section{Results}

\section{Preoperative Virtual Simulation of Skin Incision and Craniotomy}

Case 1

A 37-year-old right-handed man with a right posterior frontal LGG (Fig. 1A) was planned for tumor excision. Preoperative T2-weighted FLAIR MR images were reconstructed into 3D images using the VR technique (Fig. $1 \mathrm{C}-\mathrm{G})$. The coronal suture was marked over the skull by using the "cutting" tool (Fig. 1C), and, after adjusting the window, the marked coronal suture was visible over the lesion (Fig. 1D and F). The normal cortical surface (sulci, gyri) and morbid cortical anatomy in LGGs (expanded gyri, Fig. 1D and E) could be easily compared. The lesion's exact size was measured using the length VR tool (Fig. 1D-F). Thus, the lesion's precise measurement, both anterior and posterior to the coronal suture was appreciated (Fig. 1F). This virtual 3D image was maintained in the position the patient would assume in the operating room (Fig. 1G). Gross-total excision was achieved (Fig. 1B). This technique obviates the requirement for expensive neuronavigation systems and is especially helpful for teaching residents about surgical planning.

\section{Cortical Surface Marking and Navigation for Deep-Seated Gliomas}

Case 2

It is imperative to plan the safe entry site (corticectomy site) and shortest trajectory in deep-seated lesions. A 21-year-old right-handed female presented with persistent headache for 1 year. MRI showed a well-defined contrastenhancing left peritrigonal mass lesion (Fig. 2A), and surgical excision was planned. Since the lesion was contrast enhancing, the contrast-enhanced MRI sequence was converted into virtual 3D images using the VR technique (Fig. 2C and D). The lesion's horizontal distance from the 

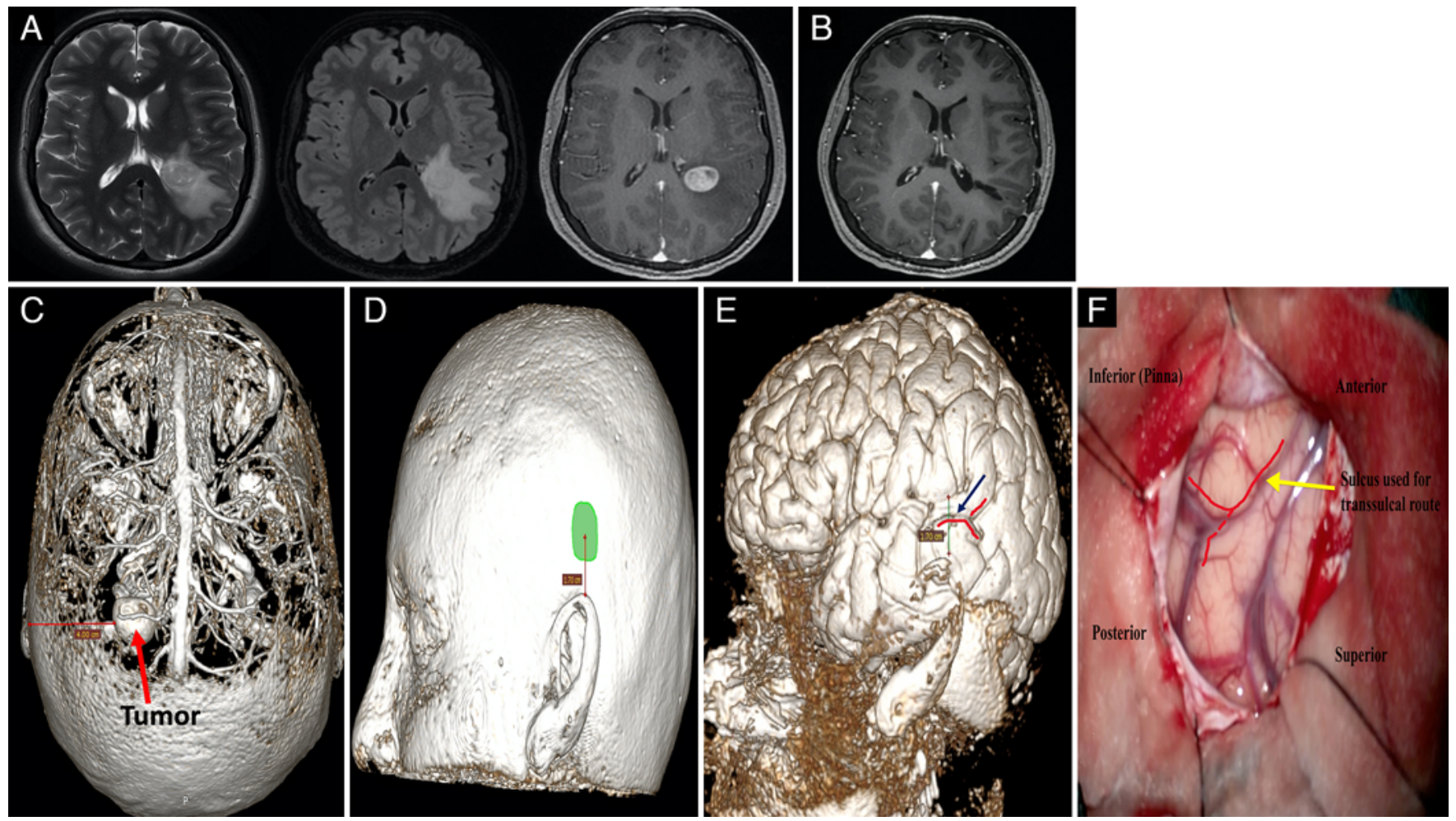

FIG. 2. Case 2. Imaging. A: Axial T2-weighted (left), FLAIR (center), and contrast (right) MR images showing a left peritrigonal mass lesion. Since the lesion was contrast enhancing, the contrast MRI sequence was converted into virtual 3D images using the VR technique. B: A transsulcal approach via this sulcus was taken to remove the tumor completely at a depth of $4 \mathrm{~cm}$, as seen in the axial MR image. Planning. C and D: The lesion's shortest horizontal distance from the surface and tumor's core from the pinna's superior border were measured. The green area in D denotes the tumor outline. E: A T2-weighted FLAIR sequence was converted to a 3D virtual image to visualize a horizontal Y-shaped sulcus overlying the tumor. F: After the dura mater was opened, the same Y-shaped sulcus was pictured. Video 1 demonstrates these steps in detail.

brain surface and that of the tumor's core from the pinna's superior border were measured (Fig. 2C and D). Then, the T2-weighted FLAIR sequence was used to reconstruct 3D virtual images to visualize the sulci and gyri (Fig. 2E). The marked horizontal Y-shaped sulcus (Fig. 2E) was planned as the entry point for the transsulcal approach. A small craniotomy above the pinna was made. After the dura mater was opened, the same horizontal sulcus with two limbs (seen on the preoperative 3D virtual image) was visualized (Fig. 2F). A transsulcal approach via this sulcus was taken to encounter the lesion at a depth of $4 \mathrm{~cm}$, and total excision (Fig. 2B) was achieved (Video 1).

\section{Preoperative 3D Morbid Cortical Surface and Venous Anatomy in Surfacing Gliomas}

Case 3

A 41-year-old right-handed male with left-sided faciobrachial sensory seizures for 1 year was diagnosed with an LGG in the right parietal lobe. Standard MR images showed a diffuse glioma with focal enhancement in the right parietal lobe (Fig. 3A). An awake parietal craniotomy and excision with intraoperative sensorimotor mapping were planned. A 3D virtual reconstruction of the FLAIR MRI sequence demonstrated the lesion morphology in 3D (Fig. 3C) and morbid surface anatomy (narrowed precen- tral gyrus pushed anteriorly, Fig. 3D). Contrast-enhanced 3D MRI reconstructed images showed the three cortical veins with respect to the lesion (Fig. 3E). Intraoperatively, an exact morbid cortical anatomy with the three cortical veins was visible (Fig. 3F and G). The smaller cortical vein (cortical vein 2, shown in Fig. 3F and G) was sacrificed while preserving the two cortical veins (cortical veins 1 and 3) at either end of the lesion. Near-total excision was possible with no deficit (Fig. 3B). Preoperative knowledge of the morbid cortical and venous anatomy in 3D helps the surgeon make a mental image and create a foolproof plan to attack the lesion.

\section{Identifying Operative Corridor Between Cortical Veins in Cortical and Subcortical Gliomas}

Case 4

A 50-year-old right-handed female diagnosed with right posterior frontal HGG was planned for excision (Fig. 4AC). As the lesion was heterogeneously contrast enhancing (Fig. 4B and C), a contrast-enhanced MRI sequence was used for creating the virtual 3D images. Virtual 3D images delineated the rounded mass lesion and two cortical veins at either end (Fig. 4D and E). After dural opening, the mass lesion with cortical veins, one each at its anterior and posterior borders, was encountered (Fig. 4F). The 

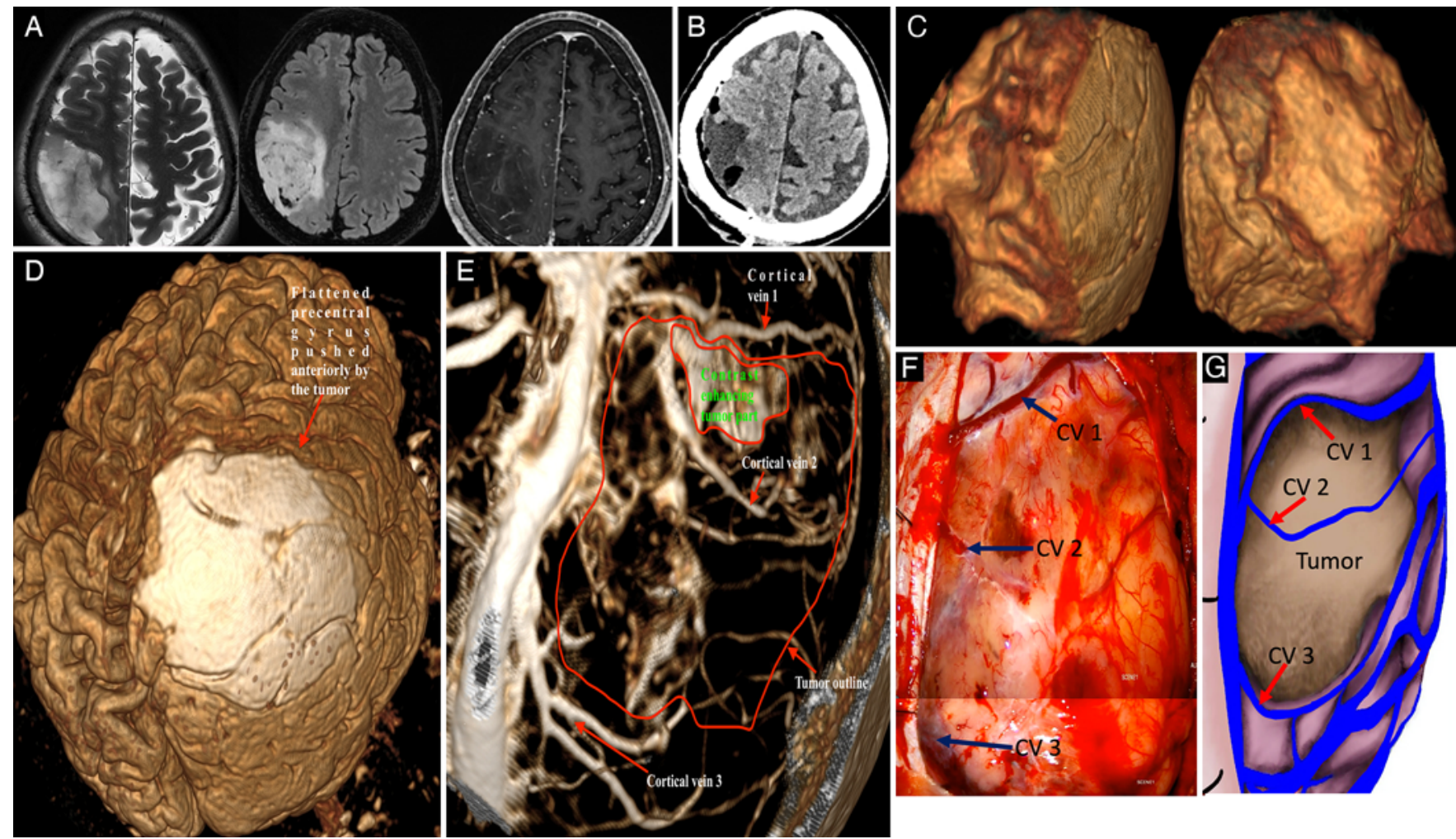

FIG. 3. Case 3. Imaging. A: Axial T2-weighted (left), FLAIR (center), and contrast MR (right) images suggestive of a right parietal lobe LGG. B: Near-total excision was done, as seen on the axial CT image. Planning. C and D: A 3D virtual reconstruction of the T2-weighted FLAIR MR image demonstrates the lesion morphology in 3D (C) and morbid surface anatomy (D). E: Contrast MRI 3D reconstructed images showed the three cortical veins with respect to the lesion. F: After intraoperative dural opening, an exact morbid cortical anatomy with the three cortical veins was visible. G: Illustration of the intraoperative photograph showing the tumor with three cortical veins. CV1 = cortical vein 1; CV2 = cortical vein 2; CV3 = cortical vein 3.

glioma was excised by working in the corridor between the veins. Both cortical veins were preserved.

\section{Case 5}

A 33-year right-handed male presented with a pineal region tumor (Fig. 5A) and was planned for surgery via a posterior transcallosal approach. A 3D virtual reconstructed image using a contrast-enhanced MRI sequence was made after removing the scalp, bone, and dura over the lesion (using the cutting tool, Fig. 5C). Thus, a virtual right parietal craniotomy (placed in the surgical position) showed the mass lesion at depth and two cortical veins draining in the superior sagittal sinus (SSS) related to the lesion (Fig. 5C). Thus, preoperatively, the trajectory to reach the lesion via a interhemispheric transcallosal approach between those two cortical veins was planned. This preoperative surgical plan was executed intraoperatively (Fig. 5D-F), aiding in reaching and excising the lesion safely (Fig. 5B).

\section{Preoperative and Postoperative 3D Visualization of Diffuse LGGs}

Case 6

A 31-year-old right-handed male with complex partial seizures was diagnosed with a left temporal LGG
(Fig. 6A). The patient underwent a left temporal craniotomy and near-total excision of the tumor (Fig. 6B). In addition to the standard FLAIR MRI sequence, 3D images were constructed using the VR technique, showing a welldefined mass lesion in three dimensions with excellent detail (Fig. 6C and D). We then generated a 3D reconstruction of tumor using postoperative FLAIR sequences (Fig. $6 \mathrm{E}$ and F) and did a volumetric analysis of the extent of resection utilizing this technique. These images helped in understanding the spatial geometry of the residual lesions.

\section{Discussion}

The 3D VR technique involves 3D reconstruction of different MRI sequences to generate high-quality 3D images that can be used for surgical planning. ${ }^{21}$ With the advancement in MRI and CT technology, it is now possible to easily reconstruct the standard $2 \mathrm{D}$ MR and CT images (available in DICOM format) into virtual 3D images..$^{21-23}$ In this case series, we report our experience and different applications of this technique in the surgical management of supratentorial gliomas, focusing mainly on the advantages of using RadiAnt software, different MRI sequences required for the virtual 3D visualization of LGGs and HGGs, and clinical utility of this technique in both surfacing and deep-seated gliomas. 

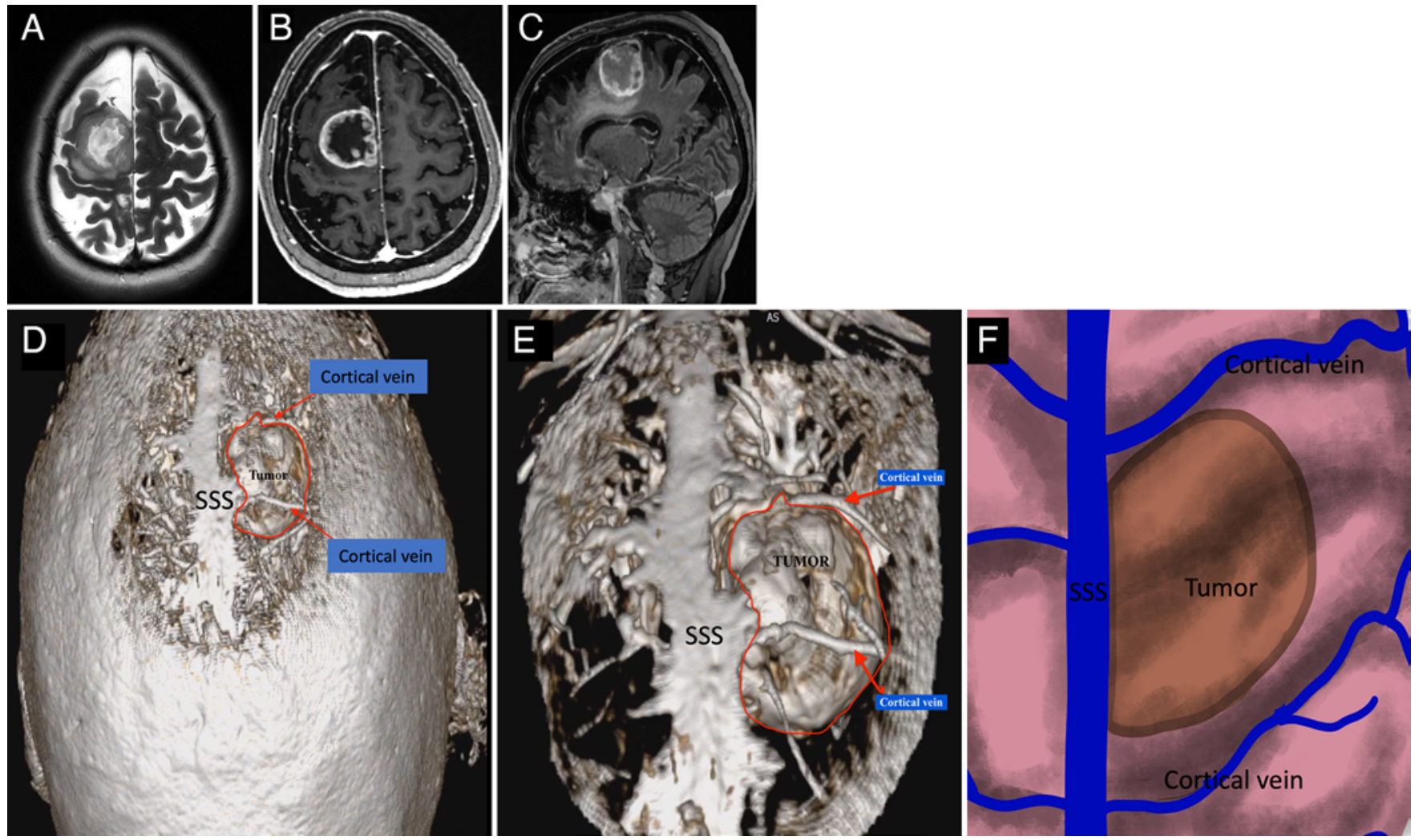

FIG. 4. Case 4. A-C: Axial T2-weighted (A) and axial (B) and sagittal $(C)$ contrast-enhanced MR images showing a surfacing right posterior frontal HGG. As the lesion was contrast-enhancing (B and C), virtual 3D images were made using the contrast sequence. D and E: Virtual 3D images delineated the rounded mass lesion and two cortical veins at either end. F: After the dura was opened, a mass lesion with a cortical vein at its anterior and posterior borders was encountered, as shown in the illustration. The lesion was excised by working in between the veins and preserving them. SSS = superior sagittal sinus.

A host of commercial as well as open-source 3D VR software programs is available online. Important ones in use include RadiAnt, Horos, and OsiriX. ${ }^{19-21,31-34}$ RadiAnt works on the Microsoft Windows operating system as well as on the Macintosh operating system while OsiriX and Horos are available for the Macintosh operating system only. RadiAnt is entirely free, as is Horos, while OsiriX is a paid software. Though OsiriX Lite (a tailored-down version of OsiriX MD), free software, is also available, it has limited basic functionality. Unlike previous studies that have mainly used OsiriX, ${ }^{, 2,23,31-34}$ ours is the first study demonstrating the VR technique in the image-guided management of gliomas using RadiAnt software. In our experience, apart from the fact that it is free, RadiAnt (unlike OsiriX and Horos) has a very user-friendly and intuitive operator interface. There is a single toolbar in RadiAnt with only a few but powerful tools that can be quickly learned, even by a person who is uncomfortable with computer-based technology. ${ }^{21}$ Furthermore, it is effortless to convert 3D images in DICOM format to stereolithography format (the standard file format for 3D printing) using RadiAnt with a single click. This process is very cumbersome in OsiriX.

In 2006, Esposito et al. reported for the first time the use of a 3D virtual reconstruction technique in the transsulcal excision of a left insular cavernoma. ${ }^{35}$ The corti- cal sulcus was used as a navigational landmark, and the authors reported a loss of accuracy for lesions at a depth greater than $2.5-3 \mathrm{~cm}$ with their technique. However, we could resect a deep-seated peritrigonal glioma (case 2) at a depth of $4 \mathrm{~cm}$ from the cortical surface safely by using our technique. This might be due to advanced MRI and better software that is currently available. Later, Harput et al., using OsiriX, proposed using the cortical veins instead of cortical sulci for navigation around the subcortical lesions. ${ }^{32}$ However, they did not evaluate this technique for surface-based tumors. In addition, the long learning curve associated with OsiriX is another issue. Apart from subcortical tumors located at depth, we have demonstrated this technique's advantage in surfacing gliomas as well.

Recently, Fernandes de Oliveira Santos et al. emphasized the importance of both the cortical surface and venous anatomy for showing the cortical projection of the subcortical lesions using Horos software. ${ }^{19}$ They insisted on using the FLAIR sequence for better visualization of the cortical surface. Our results corroborate their findings that the FLAIR sequence is better than a plain T1weighted MRI sequence (used in earlier studies ${ }^{22,28,32,35}$ ) for 3D visualization of the cortical surface (e.g., sulci, gyri). Additionally, our results show that, in addition to the cortical surface, the FLAIR sequence is also ideal for 

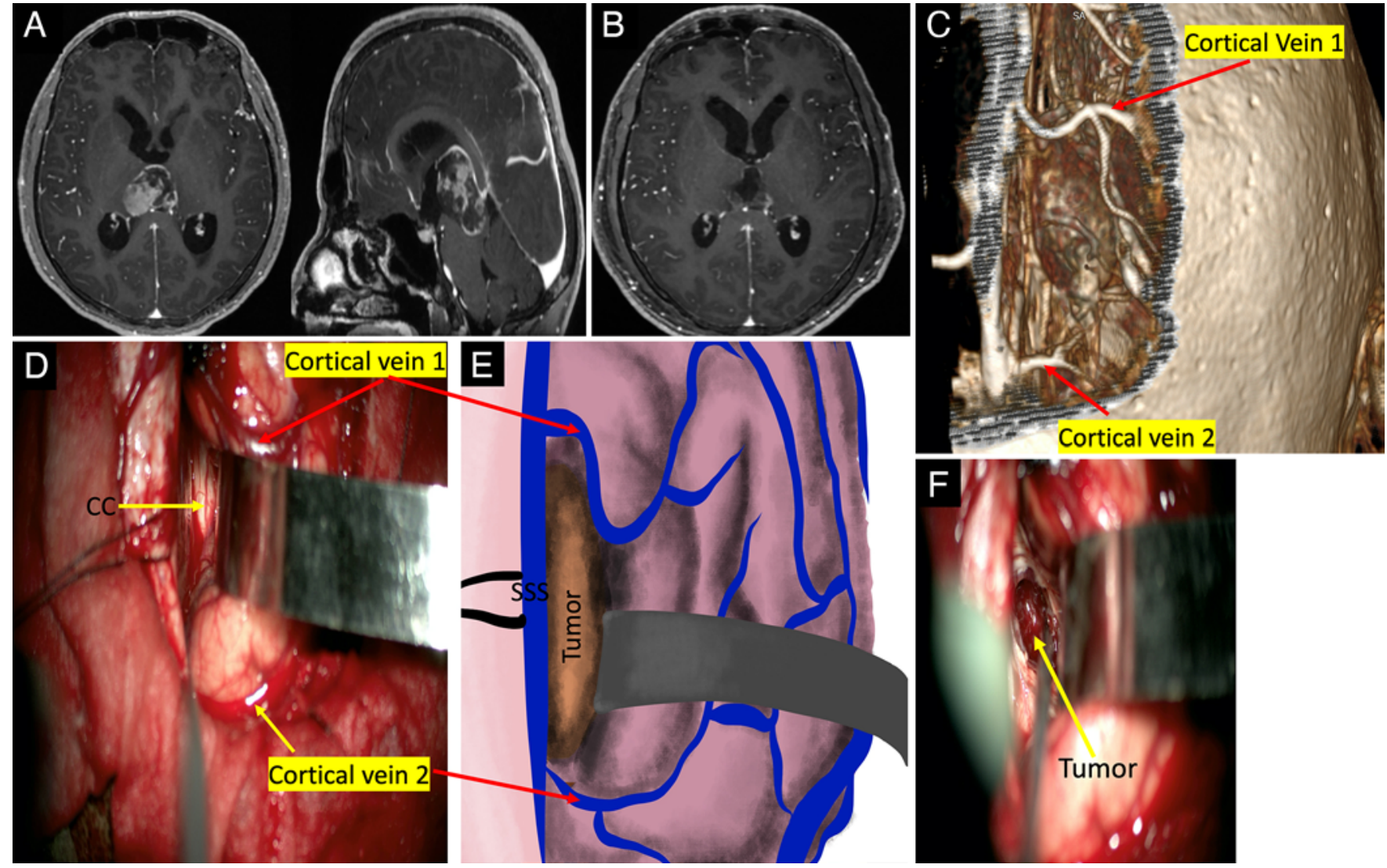

FIG. 5. Case 5. Imaging. A: Axial (left) and sagittal (right) contrast MR images showing a pineal region tumor. B: A posterior interhemispheric transcallosal route was taken between these two cortical veins to achieve near-total resection, as seen in the axial contrast MR image. Planning. C: A 3D virtual reconstructed image utilizing a contrast-enhanced MR sequence was made after removing the scalp, bone, and dura over the lesion using the cutting tool. Thus, a virtual right parietal craniotomy showed the mass lesion at depth and the two cortical veins draining in the superior sagittal sinus (related to the lesion). D-F: Intraoperative photographs $(\mathrm{D}$ and $\mathrm{F}$ ) and illustration $(\mathrm{E})$ showing the same pathoanatomy seen on the virtual $3 \mathrm{D}$ image. $\mathrm{CC}=$ corpus callosum.

3D visualization of nonenhancing diffuse LGGs (WHO grade II) by using the VR reconstruction technique. While standard MR sequences (T2-weighted and FLAIR) show these lesions as ill-defined and diffusely infiltrating the white matter, it is interesting to see these same lesions on 3D virtual images. On 3D images, these lesions show up as magnificent, well-defined spherical mass lesions, helping surgeons understand the lesion's spatial configuration (cases 3 and 6). It should be noted that these 3D virtual images of LGGs cannot be reconstructed by converting other MRI sequences, such as plain T1-weighted, T2-weighted, or contrast images. Furthermore, for 3D visualization of enhancing HGGs using this technique, contrast MRI sequences are ideal.

It is important to note that the $3 \mathrm{D}$ VR reconstruction of the postoperative T2-weighted FLAIR MRI sequence shows the extent of the lesion's resection. Compared with the standard FLAIR MRI sequence used for estimating the extent of resection in LGGs, this method might prove to be useful in the future for volumetric analysis of resection extent. In addition to helping surgeons estimate the extent of resection, preoperative and postoperative comparisons of 3D VR images of gliomas can also assist in deciphering the exact spatial location of the residual lesion in $3 \mathrm{D}$.

The 3D VR reconstruction of standard isotropically acquired submillimetric 2D MR images generates images to help the surgeon form a 3D spatial orientation of the lesion in mind. As the anatomical landmarks remain fixed even after CSF release during dural opening, navigation with cortical anatomy using this technique avoids brain shift that occurs in neuronavigation systems. ${ }^{19,32}$ Thus, this technique is invaluable in resource-constrained countries where other forms of expensive image-guided surgery tools, namely, neuronavigation systems and intraoperative MRI, are not readily available. ${ }^{20}$ Furthermore, we found the images generated by this method to be handy for teaching residents during video conferences amid the ongoing COVID-19 pandemic. ${ }^{36}$

The rapid development, availability, and application of 3D printing technology in neurosurgery has revolutionized neurosurgical teaching and planning. ${ }^{37}$ It is straightforward to convert 3D anatomical pictures into 3D models in RadiAnt software if a 3D printer is available. This 3D model can be taken to the operating room for guidance during surgery. Furthermore, integrated 3D models of diffusion 

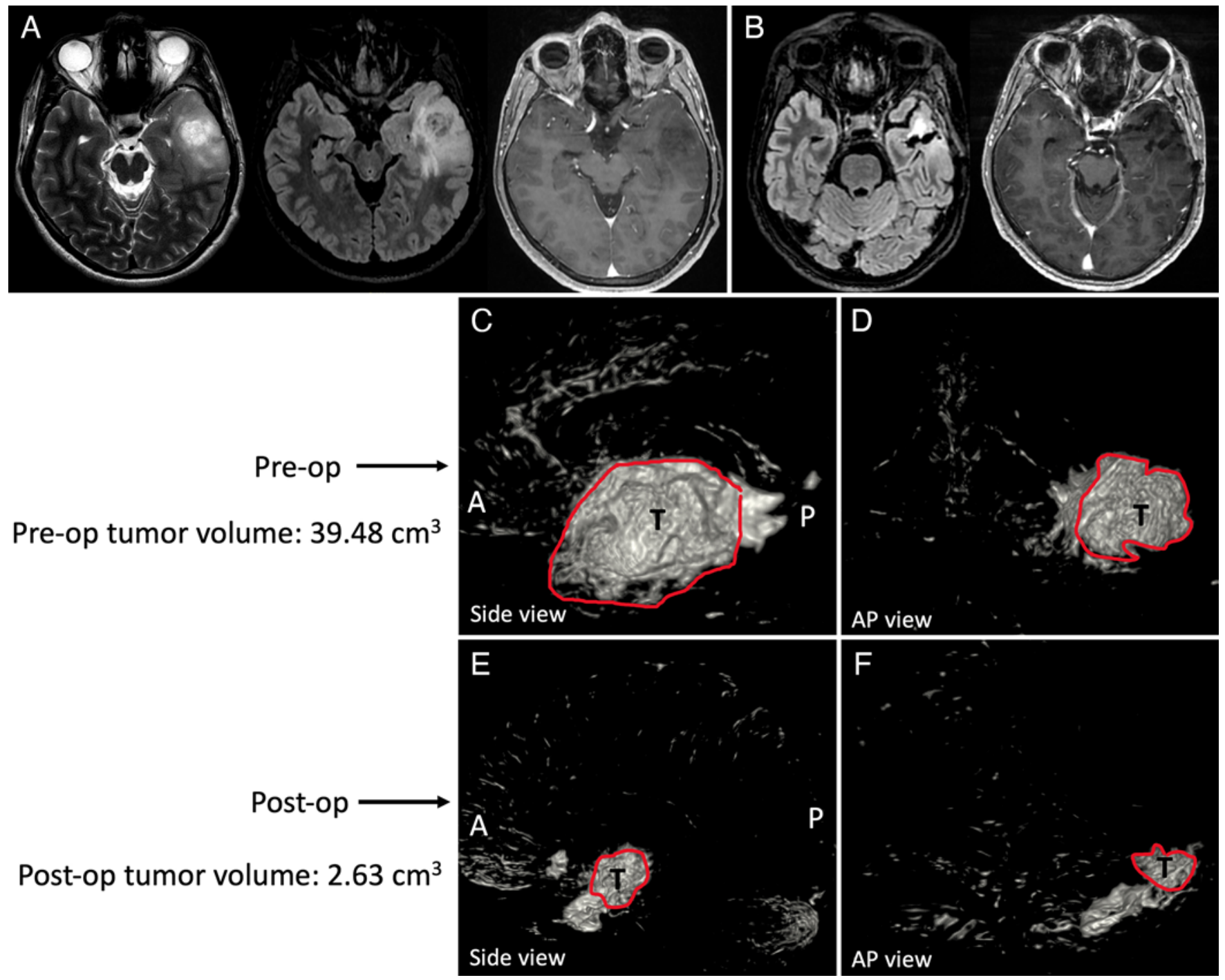

FIG. 6. Case 6. A and B: Preoperative (A) axial T2-weighted (left), FLAIR (center), and contrast (right) MR images and postoperative (B) axial FLAIR (left) and contrast (right) MR images obtained in a patient with a left temporal LGG that was near-totally excised with no new deficits. C and D: Preoperative 3D reconstructed images made using a FLAIR sequence via the VR technique showing the lesion morphology in 3D. E and F: Postoperative 3D images made from the postoperative FLAIR sequence, demonstrating spatial geometry of the residual lesion. AP = anteroposterior; $\mathrm{T}=$ tumor.

tractography images, task-based functional MR images with 3D T1-weighted/FLAIR images, could produce a patient-specific functional model for better surgical planning.

As this is a case series, we could not assess this technique's impact on outcomes such as the extent of resection and patient survival. However, we do believe that with the broader acceptance and implementation of this technique by neurosurgeons in the future, evidence regarding such outcomes might be generated.

\section{Conclusions}

The 3D VR technique using RadiAnt software is an inexpensive and easy-to-learn method of simulating glioma surgery in a virtual environment. This technique complements the available standard neuronavigation systems in image-guided neurosurgery and is especially useful in developing countries that cannot afford such expensive systems. FLAIR MRI sequences are ideal for recreating the cortical surface anatomy and the visualization of diffuse nonenhancing LGGs in 3D. Contrast MR sequences are perfect for visualizing the cortical veins and HGGs in 3D using the VR technique. Compared with the standard FLAIR MRI sequence for estimating the extent of resection in LGGs, volumetric analysis of resection extent by comparing 3D virtual reconstructions of pre- and postoperative FLAIR sequences (made using VR technique) might prove to be useful in the future.

\section{Acknowledgments}

We acknowledge Dr. Jayakumar, Pediatric Surgery Resident, 
AIIMS, Jodhpur, and Dr. Himanshi Bhati, MBBS, AIIMS Jodhpur, for helping us with the sketches.

\section{References}

1. Spiegel EA, Wycis HT, Marks M, Lee AJ. Stereotaxic apparatus for operations on the human brain. Science. 1947; 106(2754):349-350.

2. Thomas NWD, Sinclair J. Image-guided neurosurgery: history and current clinical applications. J Med Imaging Radiat Sci. 2015;46(3):331-342.

3. LeRoux PD, Berger MS, Ojemann GA, Wang K, Mack LA. Correlation of intraoperative ultrasound tumor volumes and margins with preoperative computerized tomography scans. An intraoperative method to enhance tumor resection. $J$ Neurosurg. 1989;71(5 pt 1):691-698.

4. Koivukangas J, Louhisalmi Y, Alakuijala J, Oikarinen J. Ultrasound-controlled neuronavigator-guided brain surgery. $J$ Neurosurg. 1993;79(1):36-42.

5. Watanabe E, Watanabe T, Manaka S, Mayanagi Y, Takakura $\mathrm{K}$. Three-dimensional digitizer (neuronavigator): new equipment for computed tomography-guided stereotaxic surgery. Surg Neurol. 1987;27(6):543-547.

6. Watanabe E, Mayanagi Y, Kosugi Y, Manaka S, Takakura K. Open surgery assisted by the neuronavigator, a stereotactic, articulated, sensitive arm. Neurosurgery. 1991;28(6):792-800.

7. Barnett GH, Kormos DW, Steiner CP, Weisenberger J. Intraoperative localization using an armless, frameless stereotactic wand. Technical note. J Neurosurg. 1993;78(3): 510-514.

8. Zinreich SJ, Tebo SA, Long DM, Brem H, Mattox DE, Loury $\mathrm{ME}$, et al. Frameless stereotaxic integration of $\mathrm{CT}$ imaging data: accuracy and initial applications. Radiology. 1993; 188(3):735-742.

9. Yrjänä SK, Tuominen J, Koivukangas J. Intraoperative magnetic resonance imaging in neurosurgery. Acta Radiol. 2007;48(5):540-549.

10. Ganslandt O, Behari S, Gralla J, Fahlbusch R, Nimsky C. Neuronavigation: concept, techniques and applications. Neurol India. 2002;50(3):244-255.

11. Zhang J, Chen X, Zhao Y, Wang F, Li F, Xu B. Impact of intraoperative magnetic resonance imaging and functional neuronavigation on surgical outcome in patients with gliomas involving language areas. Neurosurg Rev. 2015;38(2):319-330.

12. Roder C, Bisdas S, Ebner FH, Honegger J, Naegele T, Ernemann U, Tatagiba M. Maximizing the extent of resection and survival benefit of patients in glioblastoma surgery: highfield iMRI versus conventional and 5-ALA-assisted surgery. Eur J Surg Oncol. 2014;40(3):297-304.

13. Napolitano M, Vaz G, Lawson TM, Docquier MA, van Maanen A, Duprez T, Raftopoulos C. Glioblastoma surgery with and without intraoperative MRI at 3.0T. Neurochirurgie. 2014;60(4):143-150.

14. Senft C, Bink A, Heckelmann M, Gasser T, Seifert V. Glioma extent of resection and ultra-low-field iMRI: interim analysis of a prospective randomized trial. Acta Neurochir Suppl. 2011;109:49-53.

15. Senft C, Bink A, Franz K, Vatter H, Gasser T, Seifert V. Intraoperative MRI guidance and extent of resection in glioma surgery: a randomised, controlled trial. Lancet Oncol. 2011;12(11):997-1003

16. Willems PW, Taphoorn MJ, Burger H, Berkelbach van der Sprenkel JW, Tulleken CA. Effectiveness of neuronavigation in resecting solitary intracerebral contrast-enhancing tumors: a randomized controlled trial. J Neurosurg. 2006;104(3):360368.

17. Gerganov VM, Samii A, Akbarian A, Stieglitz L, Samii M, Fahlbusch R. Reliability of intraoperative high-resolution $2 \mathrm{D}$ ultrasound as an alternative to high-field strength
MR imaging for tumor resection control: a prospective comparative study. J Neurosurg. 2009;111(3):512-519.

18. Miner RC. Image-guided neurosurgery. J Med Imaging Radiat Sci. 2017;48(4):328-335.

19. Fernandes de Oliveira Santos B, Silva da Costa MD, Centeno RS, Cavalheiro S, Antônio de Paiva Neto M, Lawton MT, Chaddad-Neto F. Clinical application of an open-source 3D volume rendering software to neurosurgical approaches. World Neurosurg. 2018;110:e864-e872.

20. Malilay ORM, Ferraris KP, Navarro JEV. Editorial. Neurosurgical planning in a low-resource setting using free open-source three-dimensional volume-rendering software. Neurosurg Focus. 2021;50(1):E2.

21. Jha DK, Khera P, Bhaskar S, Garg M. Three-dimensional volume rendering: an underutilized tool in neurosurgery. World Neurosurg. 2019;130:485-492.

22. Lovato RM, Araujo JLV, Paiva ALC, Pesente FS, Yaltirik CK, Harput MV, Esteves Veiga JC. The use of Osirix for surgical planning using cranial measures and region of interest tools: technical note. Asian J Neurosurg. 2019;14(3): 762-766.

23. Spiriev T, Nakov V, Laleva L, Tzekov C. OsiriX software as a preoperative planning tool in cranial neurosurgery: a step-bystep guide for neurosurgical residents. Surg Neurol Int. 2017; 8:241.

24. Aydin K, Cokluk C, Kuruoglu E, Gelmez S, Diren B, Rakunt $\mathrm{C}$, Celik F. Using the magnetic resonance three-dimensional volume rendering for tissues technique in the planning of craniotomy flaps with linear scalp incision. Minim Invasive Neurosurg. 2006;49(4):189-193.

25. Beyer J, Hadwiger M, Wolfsberger S, Bühler K. High-quality multimodal volume rendering for preoperative planning of neurosurgical interventions. IEEE Trans Vis Comput Graph. 2007;13(6):1696-1703.

26. Chabrerie A, Ozlen F, Nakajima S, Leventon ME, Atsumi H, Grimson E, et al. Three-dimensional image reconstruction for low-grade glioma surgery. Neurosurg Focus. 1998;4(4):e7.

27. Wang YC, Liu YC, Hsieh TC, Lee ST, Li ML. Aneurysmal subarachnoid hemorrhage diagnosis with computed tomographic angiography and OsiriX. Acta Neurochir (Wien). 2010;152(2):263-269.

28. Gong XY, Higano S, Mugikura S, Umetsu A, Murata T, Kumabe T, Takahashi S. Virtually peeling off the skull and scalp: a simple way of mapping the superficial cerebral veins on the brain surface. Stereotact Funct Neurosurg. 2008;86(6): 345-350.

29. de Notaris M, Palma K, Serra L, Enseñat J, Alobid I, Poblete $\mathrm{J}$, et al. A three-dimensional computer-based perspective of the skull base. World Neurosurg. 2014;82(6)(suppl):S41-S48.

30. Oishi M, Fukuda M, Ishida G, Saito A, Hiraishi T, Fujii Y. Presurgical simulation with advanced 3-dimensional multifusion volumetric imaging in patients with skull base tumors. Neurosurgery. 2011; 68(1 Suppl Operative):ons188ons199.

31. Rotariu DI, Ziyad F, Budu A, Poeata I. The role of OsiriX based virtual endoscopy in planning endoscopic transsphenoidal surgery for pituitary adenoma. Turk Neurosurg. 2017;27(3):339-345.

32. Harput MV, Gonzalez-Lopez P, Türe U. Three-dimensional reconstruction of the topographical cerebral surface anatomy for presurgical planning with free OsiriX software. Neurosurgery. 2014;10(suppl 3):426-435.

33. Jaimovich SG, Guevara M, Pampin S, Jaimovich R, Gardella JL. Neurosurgical planning using OsiriX software. Article in Spanish. Surg Neurol Int. 2014;5(5)(suppl 5):S267-S271.

34. Kim G, Jung HJ, Lee HJ, Lee JS, Koo S, Chang SH. Accuracy and reliability of length measurements on threedimensional computed tomography using open-source OsiriX software. J Digit Imaging. 2012;25(4):486-491. 
35. Esposito V, Paolini S, Morace R. Resection of a left insular cavernoma aided by a simple navigational tool. Technical note. Neurosurg Focus. 2006;21(1):e16.

36. Ruparelia J, Gosal JS, Garg M, Bhaskar S, Jha DK. Challenges to neurosurgical residency training during COVID-19 pandemic: an Indian perspective. Letter. World Neurosurg. 2020;140:464-466.

37. Randazzo M, Pisapia JM, Singh N, Thawani JP. 3D printing in neurosurgery: a systematic review. Surg Neurol Int. 2016; 7(33)(suppl 33):S801-S809.

\section{Disclosures}

The authors report no conflict of interest concerning the materials or methods used in this study or the findings specified in this paper.

\section{Author Contributions}

Conception and design: Gosal, Bhaskar. Acquisition of data: Gosal, Tiwari, T Sharma, Mahal. Analysis and interpretation of data: Gosal, Tiwari, T Sharma, Garg, Mahal. Drafting the article: Gosal, T Sharma, Agrawal, Mahal, Janu. Critically revising the article: Jha, Gosal, Agrawal, Bhaskar, RK Sharma, Janu. Reviewed submitted version of manuscript: Jha, Tiwari, Agrawal, Garg, Bhaskar, RK Sharma, Janu. Approved the final version of the manuscript on behalf of all authors: Jha. Administrative/technical/material support: Jha, Tiwari, Garg, Bhaskar, RK Sharma. Study supervision: Jha, Garg.

\section{Supplemental Information \\ Videos}

Video 1. https://vimeo.com/559924822.

\section{Correspondence}

Deepak Kumar Jha: All India Institute of Medical Sciences (AIIMS), Jodhpur, Rajasthan, India.drdeepakjha@gmail.com, jhadeepak2@rediffmail.com. 